This document is the Accepted Manuscript version of a Published Work that appeared in final form in The Journal of Molecular Spectroscopy, copyright (C) Elsevier Inc., under the citation mentioned below:

Sulfur as a hydrogen bond donor in the gas phase: Rotational spectroscopic and computational study of 3-mercaptopropionic acid (c) 2019 by Weslley G.D.P.Silva, Jennifer van Wijngaarden is

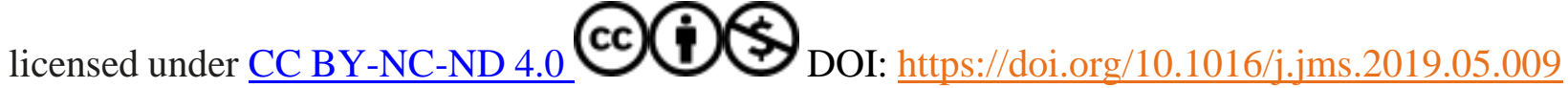

\title{
Sulfur as a hydrogen bond donor in the gas phase: Rotational spectroscopic and
} computational study of 3-mercaptopropionic acid

\author{
Weslley G. D. P. Silva ${ }^{a}$ and Jennifer van Wijngaarden ${ }^{a^{*}}$ \\ aDepartment of Chemistry, University of Manitoba, Winnipeg, Manitoba R3T 2N2, \\ Canada
}

*Corresponding author: vanwijng@umanitoba.ca 


\begin{abstract}
Rotational spectra of 3-mpa were measured from 6-18 GHz using chirped pulse and cavitybased Fourier transform microwave (FTMW) spectroscopy. Theoretical calculations using the B3LYP-D3BJ and MP2 methods with the aug-cc-pVTZ basis set show the presence of seven energy minima of 3-mpa below $8.4 \mathrm{~kJ} \mathrm{~mol}^{-1}$. Comparison of calculated rotational and centrifugal distortion constants with experimental data led to the unequivocal assignment of the global energy minimum geometry. This conformer has the mercaptan hydrogen directly oriented towards the carbonyl oxygen of the carboxylic acid moiety suggesting the presence of a six-membered ring S$\mathrm{H}^{\cdots} \mathrm{O}=\mathrm{C}$ intramolecular hydrogen bond. Quantum theory of atoms in molecules, noncovalent interactions, and natural bond orbital analyses confirm the stability of the sulfur hydrogen bond which plays a key role in the conformational preferences of 3-mpa.
\end{abstract}

Keywords: Noncovalent interactions, Hydrogen Bonds, Rotational Spectroscopy, Theoretical Calculations, Thiols 


\section{INTRODUCTION}

Hydrogen bonding $(\mathrm{HB})$ is an important noncovalent interaction which plays a central role in several processes in chemistry and biology such as organic and enzymatic catalysis, protein folding, protein structure, and molecular recognition [1]. The strength of a $\mathrm{HB} A-\mathrm{H} \cdots \mathrm{B}$ is generally proportional to the electronegativity of the $\mathrm{HB}$ donor (A) and $\mathrm{HB}$ acceptor (B) atoms. Due to the large electronegativity of oxygen $(\sim 3.4)$ and nitrogen $(\sim 3.0)$ and their versatility as both donors and acceptors, HBs formed by these atoms have been the subject of numerous studies. The characteristics of those interactions have also been used as basis for the definition of the HB concept [2]. On the other hand, less attention has been dedicated to HBs formed by atoms that have smaller electronegativities such as the chalcogens selenium $(\sim 2.5)$ and sulfur $(\sim 2.6)$ [3].

Sulfur HBs, where sulfur acts as proton donor and/or acceptor, are known to be weaker than traditional HBs; however, it was recently shown [3,4] that they can be as strong as oxygen and nitrogen $\mathrm{HBs}$ as in the case of the $\mathrm{N}-\mathrm{H} \cdots \mathrm{S}$ interaction compared to $\mathrm{N}-\mathrm{H} \cdots \mathrm{O}=\mathrm{C}$ in methionine dipeptides [3]. They are also key to the stability and function of organic crystals and biological molecules [3,5]. One example is the role of the $\mathrm{SH}^{\cdots} \mathrm{O}=\mathrm{C}$ HB in the stabilization of globular proteins in the natural environment [5]. Despite their unquestionable significance, the nature, strength, and directionality of sulfur HBs are still topics of debate in the literature [3]. The lack of information on these important interactions compromises their identification and the characterization of their function in different systems. For instance, computational algorithms which use HB geometries (distances and angles) and energetic criteria often fail to identify weak sulfur HBs in proteins since codes are parameterized with experimental data from typical and strong HBs such as the ones involving oxygen and nitrogen [2]. 
The combination of molecular spectroscopy, particularly rotational spectroscopy, and quantum mechanical calculations has been shown to be an efficient approach to overcome the lack of experimental data on weak noncovalent interactions [6-9]. FTMW spectroscopy allows the determination of precise structural and energetic parameters of molecules in a supersonic jet expansion where species are studied in isolation of crystal packing and solvent effects. Additionally, the technique provides very accurate spectroscopic parameters for comparison to values obtained from theoretical calculations making rotational spectroscopy a powerful tool for benchmark purposes [10].

A subset of sulfur-containing species has been studied in the gas phase using rotational spectroscopy to elucidate sulfur HBs [11-14]. The investigated systems are mostly dimers and molecular complexes where sulfur acts as HB donor and HB acceptor. Examples include the recent reports of Juanes et al [14] and Das et al [13] which reveal the competition between the HB donor and HB acceptor characters of sulfur in the monohydrate clusters of furfuryl mercaptan and the HB S-H ${ }^{\cdots} \mathrm{S}$ interaction in the $\mathrm{H}_{2} \mathrm{~S}$ dimer, respectively. Although some studies have been carried out, there are still several classes of sulfur $\mathrm{HBs}$ that need clarification such as the $\mathrm{S}-\mathrm{H} \cdots \mathrm{O}=\mathrm{C}$ HB which plays a key role in biological systems [5]. As recently stated by Biswal [3] and reinforced by Juanes et al [14], further investigations on unexplored sulfur-containing species at the molecular level are necessary to gain deeper insight on sulfur HBs.

Among sulfur-containing compounds, mercaptans (or thiols) (SH) are crucial in biological processes as well as in medicinal and food industries [15]. In particular, 3-mercaptopropionic acid (3-mpa) $\left[\mathrm{HS}-\mathrm{CH}_{2}-\mathrm{CH}_{2}-\mathrm{C}(=\mathrm{O}) \mathrm{OH}\right]$ is an organic compound used in the synthesis of nanocrystals [16], peptides, and protein thioesters [17], and is a convulsant and important intermediate in biological studies $[15,18]$. Due to the potential of forming important metal complexes in seawater 
and sediments, 3-mpa complexes have also been used as prototypes for theoretical investigations [19] that aim to better understand the interaction between organic thiols and metal ions. A detailed understanding of the molecular structures adopted by the 3-mpa monomer, however, is crucial to gain insight into the binding mechanisms and formation of 3-mpa-metal complexes. As the presence of a thiol $(\mathrm{SH})$ and a carboxylic acid moiety in its structure may permit formation of intramolecular $\mathrm{SH} \cdots \mathrm{O}=\mathrm{C}$ HBs, this compound is an interesting model for the study of sulfur noncovalent interactions.

Herein, we report the first microwave spectroscopic study of 3-mpa supported by density functional theory (DFT) and $a b$ initio quantum mechanical calculations. The elucidation of the conformational preferences of 3-mpa expands the current understanding of sulfur HBs and reveals important differences in comparison to its oxygen analog 3-hydroxypropionic acid.

\section{METHODS}

\section{Experimental Section}

Rotational spectra of 3-mpa were measured in the frequency range of 6-18 GHz using both chirped pulse (CP) [20] and cavity-based Balle-Flygare (BF) [21] type Fourier transform microwave (FTMW) spectrometers available at the University of Manitoba. The spectrometers have been previously described in the literature [22,23]. Initial measurements were carried out using the CP-FTMW instrument where the broadband spectra of 3-mpa were recorded in segments of $2 \mathrm{GHz}$ from $6 \mathrm{GHz}$ to $18 \mathrm{GHz}$. Afterwards, final frequency measurements were performed using the BF-FTMW instrument which provides better resolution and sensitivity. It is worth noting that in the cavity-based spectra, the rotational transitions are split into two components due to the 
Doppler effect of the molecular emission propagating collinearly with the jet expansion and have line widths of $\sim 7 \mathrm{kHz}$ (FWHM). The uncertainty in the line positions is of about $\pm 1 \mathrm{kHz}$.

For both experiments, 3-mpa (99\%), purchased from Sigma-Aldrich Canada, was used without further purification. Since the compound is a liquid at room temperature with a high boiling point $\left(110-111^{\circ} \mathrm{C}\right.$ at $\left.15 \mathrm{mmHg}\right)$, a glass bubbler was used to deliver the sample into the spectrometers with neon $(\sim 1$ bar $)$ acting as a carrier gas. The mixture containing traces of the sample and the gas was supersonically expanded into the high vacuum chambers ( $\mathrm{P} \sim 10^{-6}$ Torr) of the instruments through a pulsed nozzle (1 mm diameter orifice).

\section{Computational Details}

The presence of multiple dihedral angles in the structure of 3-mpa make its conformational equilibrium rich as multiple conformations may arise from rotations around its single bonds as shown in Figure 1. To find all possible geometries of 3-mpa, an initial conformational search was carried out using the Merck Molecular Force Field (MMFF94) [24] method at the basis of the Marvin Sketch 16.10.10 program (ChemAxon version 6.1). The search led to thirty possible geometries of 3-mpa. Optimization calculations were then carried out for all structures at the B3LYP-D3BJ [25-27] /aug-cc-pVTZ [28] level of theory. These calculations resulted in seven energy minima with relative energies smaller than $8.4 \mathrm{~kJ} \mathrm{~mol}^{-1}$. Based on recent reports [10,29] in rotational spectroscopy that highlight the importance of choosing appropriate theoretical methods for this type of study, we carried out optimization calculations at different levels of theory for the seven most stable conformers. The chosen methods include ab initio (MP2) [30] and DFT B3LYP, along with other three DFT functionals, including the 
M062-X [31], $\omega$ B97X-D [32] and the dispersion-corrected B3LYP functional with Becke-Johnson damping (B3LYP-D3BJ). The aug-cc-pVTZ basis set was used for all calculations.

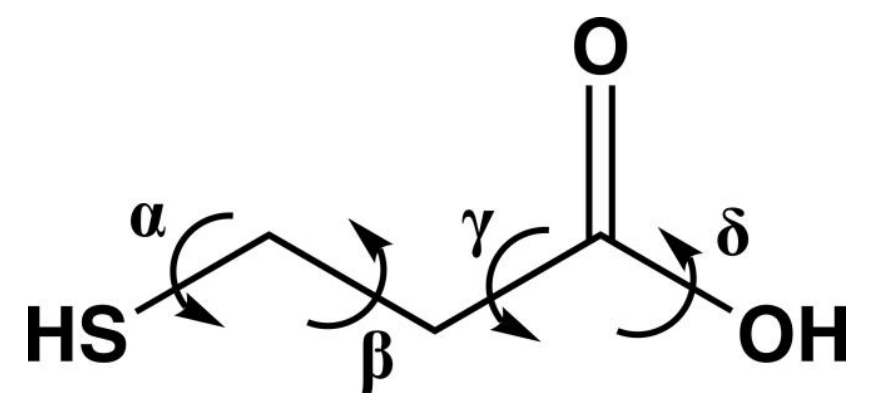

Figure 1 Chemical structure and dihedral angles of 3-mpa.

We also performed frequency calculations for all of the different methods to guarantee that imaginary frequencies were absent and also to obtain the electronic energies with zero-point corrections as well as the Gibbs free energies at 298K. All optimization and frequency calculations were carried out using the Gaussian 16 revision B.01 program [33].

Finally, to investigate the presence and stability of intramolecular interactions in the conformers of 3-mpa, we completed quantum theory of atoms in molecules (QTAIM) [34], noncovalent interactions (NCI) [35], and natural bond orbital (NBO) [36] analyses. The QTAIM, NCI, and NBO analyses were performed using the AIMAll [37], NCIPLOT [38], and NBO 6.0 [39] programs, respectively.

\section{RESULTS AND DISCUSSION}

The seven conformers of 3-mpa having relative energies less than $8.4 \mathrm{~kJ} \mathrm{~mol}^{-1}$ are shown in Figure 2 while their Cartesian coordinates are provided in Tables S1-S7 in the Supplementary 
Material file. The structures are labeled by the name "mpa" which stands for 3-mercaptopropionic acid followed by an Arabic numeral, from 1 to 7, that represents their order of stability from B3LYP-D3BJ level calculations with basis set aug-cc-pVTZ, with 1 being the most stable conformer. As conformers with higher relative energies are not appreciably populated at room temperature before supersonic expansion, only these seven are considered for further discussion.

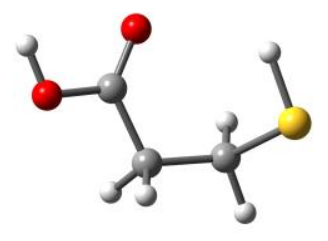

mpa1

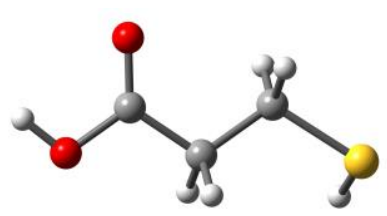

mpa2

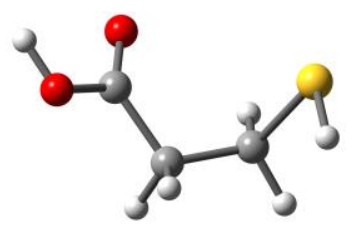

mpa3

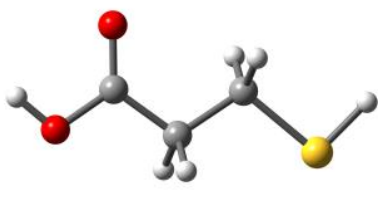

mpa4

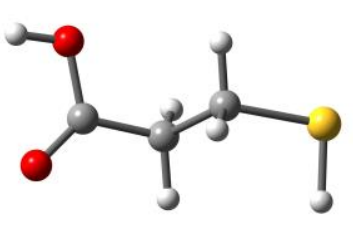

mpa5

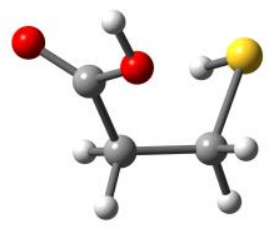

mpa6

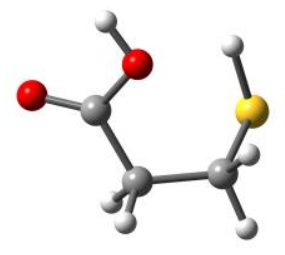

mpa7

Figure 2 Seven most stable conformers of 3-mercaptopropionic acid.

The role of theory in accurately capturing dispersive interactions in microwave spectroscopic investigations has been recently discussed in the literature [10,29]. As such, the relative energies and spectroscopic parameters of the seven most relevant conformers of 3-mpa were calculated at various levels of theory. Overall, the spectroscopic parameters calculated for each conformer from the different methods are consistent. The B3LYP-D3BJ functional presents the best agreement with the experimental data and a summary of these results is provided in Table 1 along with the ones calculated with the popular ab initio MP2 method for comparison. The results obtained using the other three DFT methods (B3LYP, M062-X, and $\omega$ B97X-D) are provided in Table S10 of the Supplementary Material. The electronic energies with zero-point corrections 
( $\left.\Delta E_{\mathrm{ZPE}}\right)$ and the Gibbs free $(\Delta G)$ energies which account for entropic contributions at $298 \mathrm{~K}$ are also provided.

Table 1 Calculated rotational parameters and relative energies (DFT B3LYP-B3BJ and MP2, aug-cc-pVTZ) for the most stable conformers of 3-mercaptopropionic acid.

\begin{tabular}{|c|c|c|c|c|c|c|}
\hline & \multicolumn{3}{|c|}{ B3LYP-D3BJ } & \multicolumn{3}{|c|}{ MP2 } \\
\hline Conformer & $A / B / C^{[\mathrm{a}]}$ & $\left|\boldsymbol{\mu}_{a}\right| /\left|\boldsymbol{\mu}_{b}\right| /\left|\boldsymbol{\mu}_{c}\right|^{[\mathrm{b}]}$ & $\Delta E_{\mathrm{ZPE}}{ }^{[\mathrm{c}]} / \Delta G^{[\mathrm{d}]}$ & $A / B / C$ & $\left|\boldsymbol{\mu}_{a}\right| /\left|\mu_{b}\right| /\left|\mu_{c}\right|$ & $\Delta E_{\mathrm{ZPE}} / \Delta G$ \\
\hline mpal & $5927 / 1572 / 1436$ & $1.6 / 1.6 / 0.1$ & $0.0 / 0.0$ & $5836 / 1618 / 1479$ & $1.7 / 1.8 / 0.1$ & $0.0 / 0.0$ \\
\hline mpa2 & $9027 / 1163 / 1049$ & $1.0 / 1.4 / 0.6$ & $3.8 / 1.7$ & $8976 / 1179 / 1062$ & $1.0 / 1.6 / 0.7$ & $4.6 / 2.5$ \\
\hline mpa3 & $5395 / 1559 / 1517$ & $0.6 / 2.4 / 1.0$ & $4.6 / 3.4$ & $5277 / 1621 / 1577$ & $0.5 / 2.6 / 1.3$ & $4.6 / 3.3$ \\
\hline mpa4 & $8972 / 1183 / 1058$ & $0.6 / 0.4 / 0.0$ & $6.3 / 4.2$ & $8917 / 1200 / 1072$ & $0.5 / 0.4 / 0.0$ & $6.7 / 4.6$ \\
\hline mpa5 & $8394 / 1181 / 1092$ & $0.2 / 0.8 / 0.0$ & $7.9 / 5.0$ & $8338 / 1199 / 1108$ & $0.1 / 0.8 / 0.0$ & $8.4 / 5.0$ \\
\hline mpa6 & 4904/1662/1627 & $0.4 / 2.0 / 0.8$ & $7.1 / 6.3$ & $4817 / 1738 / 1686$ & $0.5 / 2.4 / 0.5$ & $6.3 / 5.9$ \\
\hline mpa7 & $5231 / 1618 / 1554$ & $0.0 / 0.5 / 2.2$ & $7.1 / 6.3$ & $5087 / 1679 / 1626$ & $0.1 / 0.9 / 2.4$ & $6.3 / 5.9$ \\
\hline
\end{tabular}

${ }^{[a]}$ Rotational constants $(A, B$, and $C)$ in $\mathrm{MHz} ;{ }^{[b]}$ Absolute values of the electric dipole moment components in $\mathrm{D}$; ${ }^{[c]}$ Relative energies, in $\mathrm{kJ} \mathrm{mol}^{-1}$, with respect to the global energy minimum accounting for zero-point energy (ZPE) corrections; ${ }^{[\mathrm{d}]}$ Relative Gibbs free energies, in $\mathrm{kJ} \mathrm{mol}^{-1}$, with respect to the global energy minimum calculated at $298 \mathrm{~K}$.

From both the B3LYP-D3BJ and MP2 methods, conformer mpa1 is predicted to be the geometry corresponding to the global energy minimum and to be at least $1.7 \mathrm{~kJ} \mathrm{~mol}^{-1}(\Delta G$; B3LYP-D3BJ/aug-cc-pVTZ) more stable than the second lowest energy conformer (mpa2). This energy difference corresponds to abundances that are equivalent to $44.9 \%$ and $21.2 \%$ for mpa1 and mpa2 respectively, at room temperature. In the most stable conformer, the hydrogen atom of the SH group is directly oriented towards the carbonyl oxygen of the carboxylic acid moiety 
suggesting the presence of an $\mathrm{S}-\mathrm{H}^{\cdots} \mathrm{O}=\mathrm{C}$ intramolecular hydrogen bond (IHB). This interaction would result in the formation of a six-membered ring ( $\beta$ of approximately $70^{\circ}$ ) in this conformation. The second lowest energy conformer adopts a "zig-zag" structure $\left(\beta \sim 180^{\circ}\right)$ where the oxygen, sulfur, and carbon atoms are all in the same plane while the hydrogens have out-of-plane orientations with the exception of the carboxylic acid hydrogen. The remaining higher energy conformations also have values of $\beta$ close to $180^{\circ}$ (mpa4 and $\left.\mathbf{m p a 5}\right)$ or $70^{\circ}(\mathbf{m p a 3}, \mathbf{m p a 6}$, and mpa7). The geometry adopted by the global energy minimum of 3-mpa is similar to the one reported in the literature for the most stable conformer of thioglycolic or mercaptoacetic acid $\left(\mathrm{HO}-\mathrm{C}(=\mathrm{O})-\mathrm{CH}_{2}-\mathrm{SH}\right)$ where the presence of the $\mathrm{S}-\mathrm{H} \cdots \mathrm{O}=\mathrm{C}$ intramolecular interaction results in the formation of a five-membered ring [40,41].

Interestingly, none of the most populated conformers of 3-mpa exhibit a favorable geometry for the formation of a $\mathrm{O}-\mathrm{H}^{\cdots} \mathrm{S}$ IHB between the sulfur and the hydroxyl group of the carboxylic acid. This reveals that the character of sulfur in 3-mpa is that of a HB donor rather than acceptor. In the computational study of the oxygen analog of 3-mpa, 3-hydroxypropionic acid, the alcohol oxygen serves as a HB donor in both of the two lowest energy conformers. The lowest energy is analogous to that determined for mpa1 in this work and the second most stable conformer of 3-hydroxypropionic acid exhibits a $\mathrm{O}-\mathrm{H}^{\cdots} \mathrm{O}$ interaction with the alcohol oxygen acting as a $\mathrm{HB}$ donor to the hydroxy oxygen of the carboxylic acid moiety [42]. The latter resembles the structure of conformer mpa6, however, in 3-mpa this geometry is at least $5.9 \mathrm{~kJ} \mathrm{~mol}^{-1}$ higher in energy than mpa1 and is thus, considerably less stable.

As there are no previous reports on the microwave spectra of 3-mpa, the data collected in Table 1 were used to guide our experimental search. As the intensity of the rotational transitions in the microwave spectra is directly related to the electric dipole moment components $\left(\mu_{a}, \mu_{b}\right.$, and 
$\left.\mu_{c}\right)$ along the principal axes $(a, b$, and $c)$ and the population of the ground state rotational energy levels, our spectral search focused first on the assignment of rotational transitions corresponding to the minimum-energy geometry. The simulated spectrum of mpa1 was compared to the experimental CP-FTMW spectra using the PGOPHER program [43]. The comparison led to the successful identification of mpa1 in the range from $6 \mathrm{GHz}$ to $18 \mathrm{GHz}$. Both $a$ - and $b$-type rotational transitions were readily detected in good agreement with the sizeable $\mu_{a}=1.6 \mathrm{D}-1.7 \mathrm{D}$ and $\mu_{b}=$ 1.6 D - 1.8 D (B3LYP-D3BJ and MP2, aug-cc-pVTZ) dipole moment components predicted by theory. Despite accurate line positions, no $c$-type transitions could be observed for mpal which also agrees with the small predicted values of $\mu_{c}=0.1 \mathrm{D}$ (B3LYP-D3BJ and MP2, aug-cc-pVTZ). A portion of the broadband microwave spectrum highlighting some rotational transitions assigned for mpa1 is provided in Figure 3A. To achieve higher resolution and sensitivity, the rotational transitions observed for the parent species of mpa1 in the broadband spectra were re-investigated using our cavity-based BF-FTMW spectrometer. In total, 45 Doppler doublets, including $26 a$-type and $19 b$-type rotational transitions, were measured for the parent species of mpa1. A sample of the BF-FTMW spectrum showing the Doppler splitting for the $33_{03}-202$ rotational transition is depicted in Figure 3B. The full list of assigned transitions is provided in Table S11 in the Supplementary Material. The observed transitions were fitted using Pickett's SPFIT program [44] set to Watson's A-reduced Hamiltonian [45] in the $I^{r}$ representation. The derived experimental parameters are shown in Table 2. 


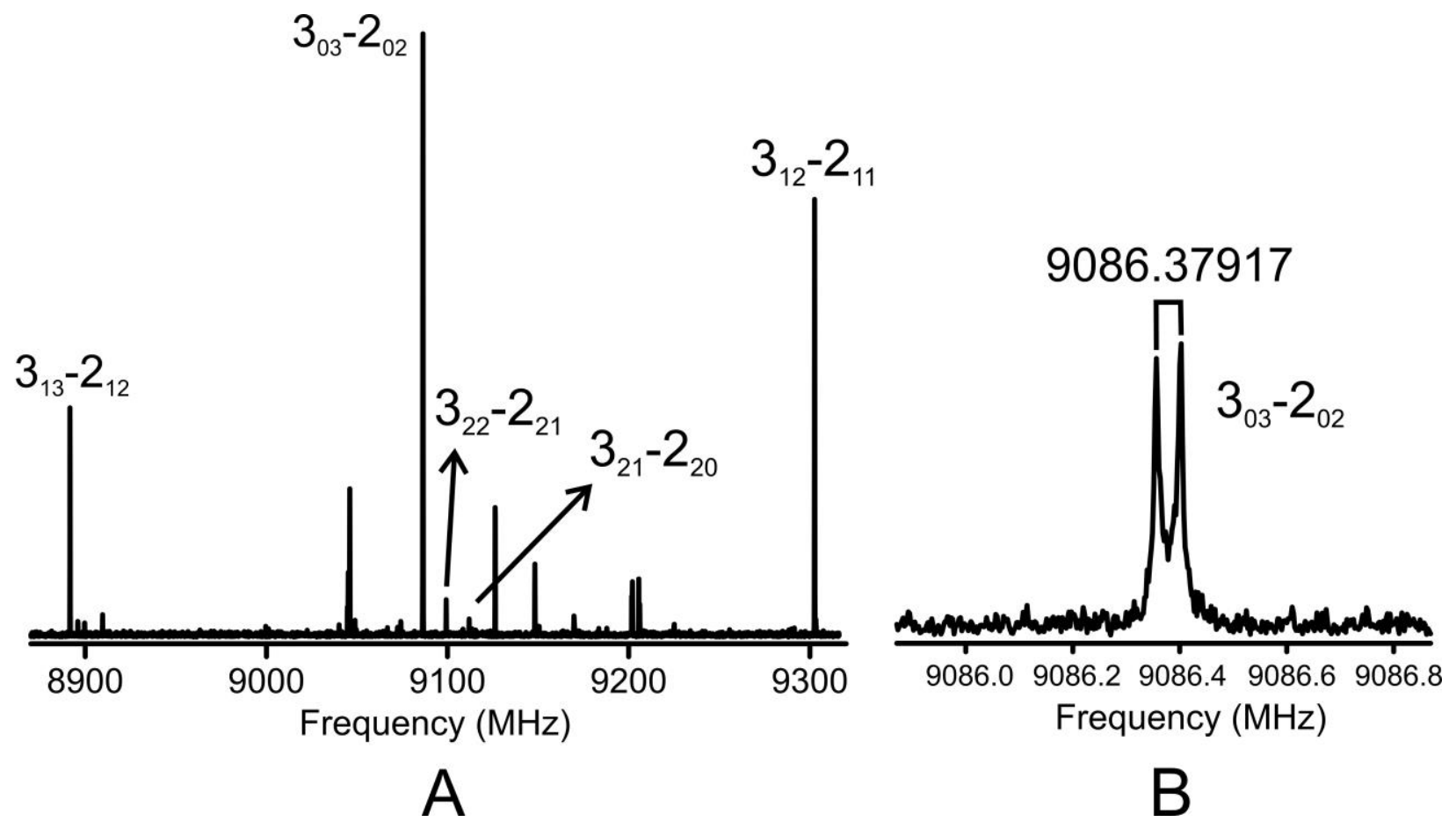

Figure 3 A) Portion of the broadband spectrum (1.5 million FIDs) of 3-mercaptopropionic acid displaying some rotational transitions observed for the parent species of mpa1; B) Sample of the cavity-based FTMW spectrum (778 cycles) of the $3_{03}-2_{02}$ rotational transition of mpa1. 
Table 2 Comparison between experimental and theoretical (B3LYP-D3BJ/aug-cc-pVTZ) spectroscopic parameters for the parent species of conformer mpa1. The calculated distortion constants were obtained through anharmonic frequency calculations.

\begin{tabular}{ccc}
\hline Parameter & Experimental & B3LYP-D3BJ \\
\hline$A / \mathrm{MHz}$ & $5946.15216(36)$ & 5927 \\
$B / \mathrm{MHz}$ & $1585.04375(10)$ & 1572 \\
$C / \mathrm{MHz}$ & $1448.00927(11)$ & 1436 \\
$\Delta_{J} / \mathrm{kHz}$ & $0.77709(61)$ & 0.77 \\
$\Delta_{J K} / \mathrm{kHz}$ & $-5.7705(39)$ & -5.59 \\
$\Delta_{K} / \mathrm{kHz}$ & $33.179(72)$ & 35.06 \\
$\delta_{j} / \mathrm{kHz}$ & $-0.03159(51)$ & -0.03 \\
$\delta_{k} / \mathrm{kHz}$ & $5.730(45)$ & 6.24 \\
$\sigma / \mathrm{kHz}$ & 0.5 & \\
$N$ & 45 & \\
\hline
\end{tabular}

The ground state experimental rotational parameters are well-determined $(\sigma=0.5 \mathrm{kHz})$ and present good agreement with the equilibrium values derived from optimization and frequency calculations. The theoretical centrifugal distortion constants were estimated only using the B3LYP-D3BJ method due to the large computational cost of anharmonic frequency calculations at the MP2 level. The experimentally-derived rotational constants $A, B$, and $C$ show better agreement with the values calculated using B3LYP-D3BJ (within 1.5\% for all rotational constants) which suggests that the equilibrium geometry $\left(r_{\mathrm{e}}\right)$ of mpa1 obtained at this level of theory is closer to its ground state structure. Comparison between experimental and theoretical centrifugal distortion constants provides an additional check for the unequivocal assignment of these transitions as arising from mpa1. It is worth noting that although a theoretical method can provide reasonable results for rotational constants, it does not always reproduce the values of the centrifugal distortion constants well [46]. Thus, the excellent match between experiment and theory in this work validates the consistency of the chosen level of theory. 
Despite our experimental efforts, no other conformers of 3-mpa could be identified in the rotational spectra. Although mpa2 is predicted to have a population between $6.4 \%$ and $33.6 \%$ at room temperature (for different levels of theory), i.e. the predicted conformer distributions are very sensitive to the computation employed, the observed transitions of 3-mpa were extremely weak due to the compound's low volatility. This prevented the identification of the spectrum of mpa2 along with those of other conformers and minor isotopologues. Furthermore, it is well known that higher energy conformers may relax to lower energy forms during the supersonic jet expansion depending on the barriers to re-arrangement. Barriers above $\sim 400 \mathrm{~cm}^{-1}$ restrict this relaxation and allow experimental observation of metastable conformers [47]. In this case, as the relaxation of mpa2 to mpa1 involves changes in all dihedral angles, the pathway is not simple to estimate, and we cannot conclude whether the absence of mpa2 is due to its low population (maybe as low as $6.4 \%$ ) or facile relaxation to mpa1 in the cold jet.

To investigate the presence and stability of the six-membered ring $\mathrm{S}-\mathrm{H} \cdots \mathrm{O}=\mathrm{C}$ IHB in conformer mpa1, we performed quantum theory of atoms in molecules (QTAIM), noncovalent interactions (NCI), and natural bond orbital (NBO) analyses which are common approaches for the characterization of hydrogen bonds and other noncovalent interactions [34-36]. The QTAIM molecular graph (Figure 4A) exhibits a bond path (BP) and a bond critical point (BCP) between the carbonyl oxygen of the carboxylic acid moiety and the mercaptan hydrogen, supporting the existence of a $\mathrm{S}-\mathrm{H}^{\cdots} \mathrm{O}=\mathrm{C}$ IHB in mpa1. Due to the formation of a six-membered ring, a ring critical point (RCP) is also observed in the QTAIM molecular graph. To confirm the stability of this interaction, the electron density $(\rho)$, its Laplacian $\left(\nabla^{2} \rho\right)$, and the local potential energy (V) at the BCP were analyzed using Bader's [48] criteria for the characterization of hydrogen bonds. The criteria establish that hydrogen bonds have values of $\rho(r)$ and $\nabla^{2} \rho(r)$ at the BCP between 0.002 a.u. 
- 0.034 a.u. and 0.024 a.u. - 0.139 a.u., respectively [49]. The values obtained for the QTAIM parameters (Table 3) at the $\mathrm{BCP}$ of the $\mathrm{S}-\mathrm{H} \cdots \mathrm{O}=\mathrm{C}$ interaction satisfy Bader's rules. Additionally, we also evaluated other criteria, established by Koch and Popelier [49] which compare the QTAIM properties of a H-bound and a non-H-bound hydrogen atom; these properties are the atomic charge $q(\mathrm{H})$, atomic first dipole moment $M_{l}(\mathrm{H})$, atomic energy $E(\mathrm{H})$, and atomic volume $V(\mathrm{H})$. By comparing the QTAIM parameters (Table 3) of the mercaptan hydrogens of mpal (which is involved in the IHB) and mpa2 (which does not experience the intramolecular interaction), it can be seen that the mercaptan hydrogen in the former has a larger $q(\mathrm{H})$, has smaller $M_{l}(\mathrm{H})$ and $V(\mathrm{H})$, and is less stable than in the latter. Thus, as the mercaptan hydrogen of mpa1 satisfies the Koch and Popelier criteria, this conformer possesses a stable $\mathrm{S}-\mathrm{H} \cdots \mathrm{O}=\mathrm{C}$ IHB which indeed plays a significant role in the stabilization of mpa1 relative to the other forms. 

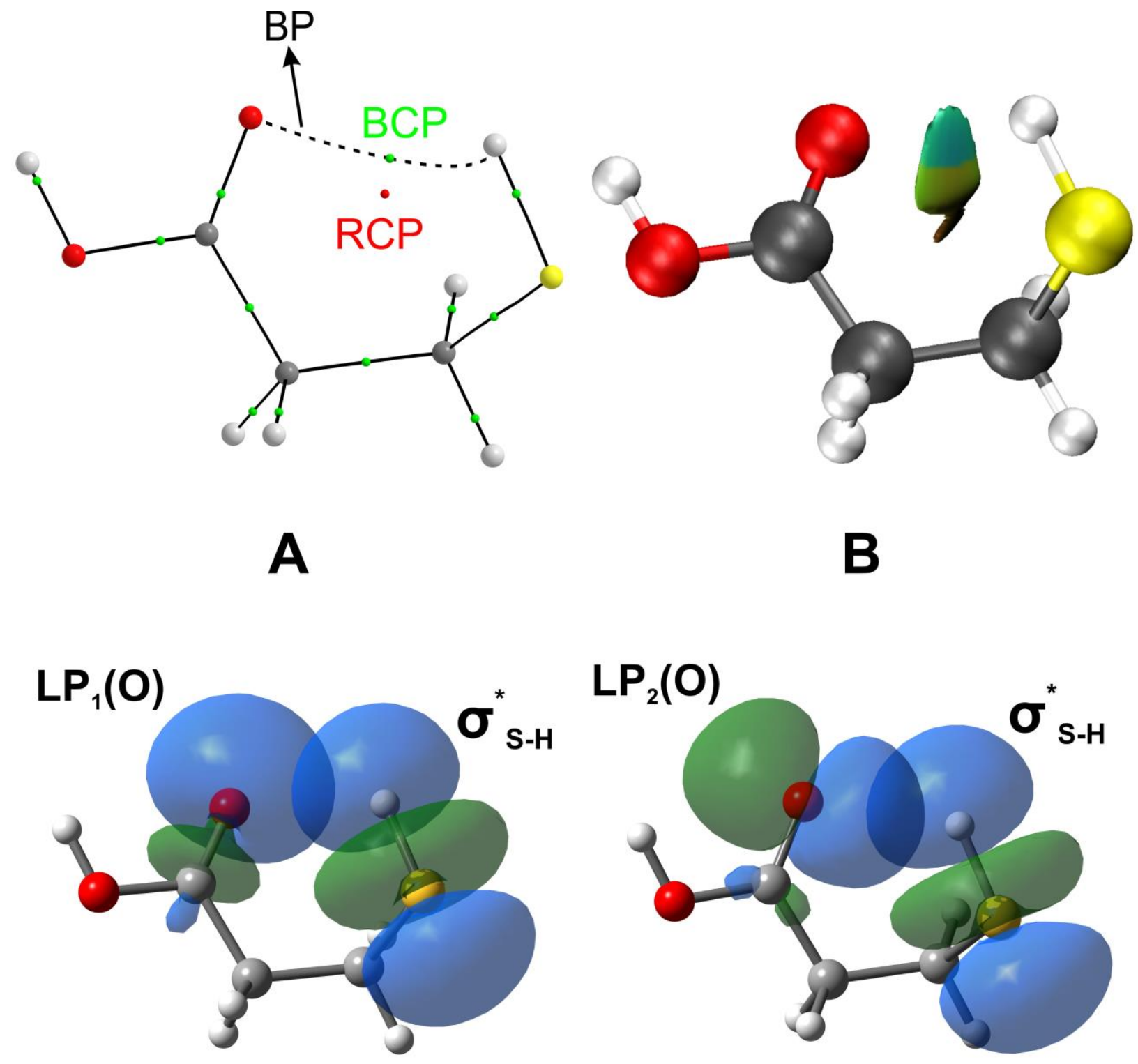

\section{$\mathrm{LP}_{2}(0)$}

B

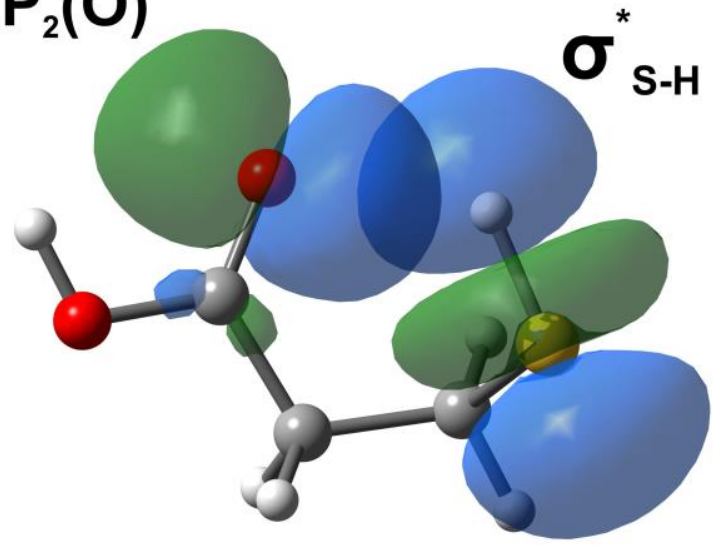

C

Figure 4 A) QTAIM molecular graph, B) NCI isosurface ( $s=0.5$ au; colour scale of $-0.02<\rho<$ 0.02); and C) NBO plots of mpa1. 
Table 3. QTAIM parameters (in a.u.) obtained for conformers mpa1 and mpa2.

\begin{tabular}{ccc}
\hline Parameter & mpa1 & mpa2 (reference) \\
\hline $\boldsymbol{\rho}(\mathbf{r})$ & 0.009 & - \\
$\boldsymbol{\nabla}^{\mathbf{2}} \mathbf{\rho}(\mathbf{r})$ & 0.033 & - \\
$\mathbf{V}(\mathbf{r})$ & -0.005 & - \\
& $\mathbf{H}(\mathbf{S H})$ & $\mathbf{H}(\mathbf{S H})$ \\
$\boldsymbol{q}(\mathbf{H})$ & -0.028 & -0.069 \\
$\boldsymbol{M}_{\boldsymbol{I}}(\mathbf{H})$ & 0.053 & 0.063 \\
$\boldsymbol{E}(\mathbf{H})$ & -0.607 & -0.620 \\
$\boldsymbol{V}(\mathbf{H})$ & 49.06 & 54.36 \\
\hline
\end{tabular}

In agreement with the QTAIM analysis, the NCI approach (Figure 4B) confirms the presence of a weak $\mathrm{S}-\mathrm{H} \cdots \mathrm{O}=\mathrm{C}$ IHB in mpa1 characterized by the blue-greenish isosurface between the carbonyl oxygen and the mercaptan hydrogen. The NCI isosurface is coloured based on the values of $\operatorname{sign}\left(\lambda_{2}\right) \rho$, where $\lambda_{2}$ is the second eigenvalue of the Hessian matrix of $\rho$. The colours are indicative of the types of interactions that are present in the chemical system under investigation where strong attractive, strong repulsive, and weak (van der Waals) interactions are represented by blue, red, and green isosurfaces, respectively.

The $\mathrm{S}-\mathrm{H} \cdots \mathrm{O}=\mathrm{C}$ IHB was also evaluated through natural bond orbital (NBO) analysis carried out at the B3LYP-D3BJ/aug-cc-pVTZ level of theory. The NBO output reveals charge transfer from the two lone-pairs of the carbonyl oxygen $\left[\mathrm{LP}_{1}(\mathrm{O})\right.$ and $\left.\mathrm{LP}_{2}(\mathrm{O})\right]$ to the anti-bonding orbital of the S-H bond $\left(\sigma^{*} \mathrm{~S}-\mathrm{H}\right)$, characterized by the $\mathrm{LP}_{1}(\mathrm{O}) \rightarrow \sigma^{*} \mathrm{~S}-\mathrm{H}$ and $\mathrm{LP}_{2}(\mathrm{O}) \rightarrow \sigma^{*} \mathrm{~S}-\mathrm{H}$ hyperconjugative interactions. The second-order perturbation energies for such interactions are equal to $0.38 \mathrm{~kJ} \mathrm{~mol}^{-1}$ and $1.55 \mathrm{~kJ} \mathrm{~mol}^{-1}$, respectively which implies greater involvement of $\mathrm{LP}_{2}(\mathrm{O})$ in the $\mathrm{S}-\mathrm{H} \cdots \mathrm{O}=\mathrm{C}$ IHB. For comparison, in 3-hydroxypropionic acid, the second-order perturbation energies of $\mathrm{LP}_{1}(\mathrm{O}) \rightarrow \sigma^{*} \mathrm{O}-\mathrm{H}$ and $\mathrm{LP}_{2}(\mathrm{O}) \rightarrow \sigma^{*} \mathrm{O}-\mathrm{H}$ are equivalent to $1.17 \mathrm{~kJ} \mathrm{~mol}^{-1}$ and $7.07 \mathrm{~kJ} \mathrm{~mol}^{-}$ ${ }^{1}$ (B3LYP-D3BJ/aug-cc-pVTZ) (this work), respectively which reveals that the energies of the hyperconjugative interactions involved in the $\mathrm{O}-\mathrm{H}^{\cdots} \mathrm{O}=\mathrm{C}$ IHB are approximately four times larger 
than the ones in the $\mathrm{S}-\mathrm{H} \cdots \mathrm{O}=\mathrm{C}$ IHB. This is of no surprise since oxygen often forms stronger hydrogen bonds when compared to sulfur. The $\mathrm{LP}(\mathrm{O}) \rightarrow \sigma^{*}$ s-H hyperconjugative interactions play a key role in the stabilization of mpa1 which explains why it is the dominant conformer in the rich conformational landscape. A pictorial representation of the orbital overlaps of the $\mathrm{LP}(\mathrm{O}) \rightarrow \sigma^{*} \mathrm{~S}-\mathrm{H}$ hyperconjugative interactions is provided in the NBO plots of Figure 4C.

Regardless of whether sulfur acts as a HB donor or HB acceptor, it has been shown that sulfur HBs are characterized by different geometric parameters when compared to the ones formed by oxygen and nitrogen [2]. Thus, apart from quantum mechanical approaches, we also determined the effective geometric parameters of the $\mathrm{S}-\mathrm{H}^{\cdots} \mathrm{O}=\mathrm{C}$ six-membered IHB by looking at the equilibrium structure $\left(r_{\mathrm{e}}\right)$ of mpa1 (Figure 5). The length of the $\mathrm{S}-\mathrm{H}^{\cdots} \mathrm{O}=\mathrm{C}$ IHB determined by the distance between the carbonyl oxygen and the mercaptan hydrogen is $2.553 \AA$ which is smaller than the sum of the van der Waals radii of oxygen and hydrogen $(\sim 2.72 \AA)$. The $\angle \mathrm{SH} \cdots \mathrm{O}$ angle is found to be $117.5^{\circ}$. For comparison, sulfur HBs in cysteine, where sulfur acts as proton donor $\mathrm{S}-\mathrm{H}^{\cdots} \mathrm{B}$, have bond lengths that vary from $2.51 \AA$ to $2.84 \AA$ depending on the nature of the HB acceptor atom B [2].

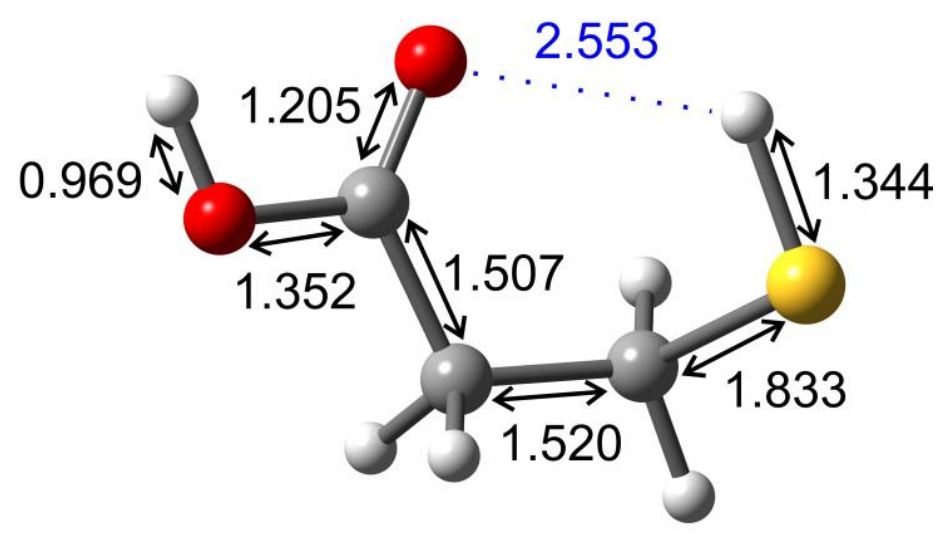

Figure 5 Equilibrium $r_{\mathrm{e}}$ structure of mpa1 obtained at the B3LYP-D3BJ/aug-cc-pVTZ level of theory. 
In previous rotational spectroscopy studies [40,41] of thioglycolic acid $\left(\mathrm{HO}-\mathrm{C}(=\mathrm{O})-\mathrm{CH}_{2}-\mathrm{SH}\right)$, which is the shorter hydrocarbon chain version of 3-mpa, the existence of a five-membered ring $\mathrm{S}-\mathrm{H}^{\cdots} \mathrm{O}=\mathrm{C}$ IHB was suggested. Interestingly, splitting related to the torsion of the $\mathrm{SH}$ group was observed in the rotational spectra by the authors. This tunneling motion is characterized by a low-energy barrier double-well potential function that allows for the interconversion between two equivalent forms of thioglycolic acid. In the present study, we did not observe splitting associated with this tunneling motion in the rotational spectra of 3-mpa which we purport is related to a stronger $\mathrm{S}-\mathrm{H}^{\cdots} \mathrm{O}=\mathrm{C}$ IHB in this compound. This leads to an increase in the energy barrier between the two equivalent forms of mpal restricting the torsion motion of the SH group in this conformation. To support our assertion, we optimized and performed QTAIM and NBO (B3LYP-D3BJ/aug-cc-pVTZ) analyses for the geometry of the previous reported conformer of thioglycolic acid. In the QTAIM molecular graph, neither a BP nor a BCP were observed regarding the $\mathrm{S}-\mathrm{H} \cdots \mathrm{O}=\mathrm{C}$ IHB indicating the absence or the weak character of this interaction in thioglycolic acid. NBO calculations confirm that the $\mathrm{S}-\mathrm{H} \cdots \mathrm{O}=\mathrm{C}$ interaction in thioglycolic acid is weaker when compared to the one in 3-mpa. This is evidenced by the calculated second-order perturbation energies of the $\mathrm{LP}_{2}(\mathrm{O}) \rightarrow \sigma^{*} \mathrm{~S}-\mathrm{H}$ hyperconjugative interaction which is equal to $0.21 \mathrm{~kJ} \mathrm{~mol}^{-1}$ in the former and $1.63 \mathrm{~kJ} \mathrm{~mol}^{-1}$ in the latter. Furthermore, it is well known that six-membered ring IHBs are stronger than the five-membered ones. This is explained by a greater ring strain in the five-membered cycles that affects the stability of the IHB formed as exemplified by the comparison of the $\mathrm{O}-\mathrm{H}^{\cdots} \mathrm{O}$ IHB in a series of substituted diols named 1,2-ethanediol [50], 1,3-propanediol [51], and 1,4-butanediol [52] that form five-, six-, and sevenmembered ring IHBs, respectively. 


\section{CONCLUSIONS}

In summary, the combination of quantum mechanical calculations and rotational spectroscopy in a supersonic jet expansion allowed the conformational study of 3-mpa for the first time. Theoretical calculations at both B3LYP-D3BJ and MP2 levels with the aug-cc-pVTZ basis set suggest the presence of seven populated energy minima in the conformational equilibrium of 3-mpa. Experimentally, one conformer was observed in the pure rotational spectra and the derived experimental rotational parameters agree very well with those predicted for the global energy minimum (mpa1) geometry whereby the sulfur behaves as a proton donor rather than acceptor. The excellent match of the experimental rotational and centrifugal distortion constants with the ones calculated at the B3LYP-D3BJ/aug-cc-pVTZ not only unequivocally confirms the assignment of mpa1 but also reveals that its actual geometry is very close to the equilibrium structure $r_{\mathrm{e}}$ obtained at this level of theory. Topological NCI and QTAIM analyses show the presence of a stable $\mathrm{S}-\mathrm{H}^{\cdots} \mathrm{O}=\mathrm{C}$ IHB in mpa1 which is confirmed by hyperconjugative $\mathrm{LP}(\mathrm{O}) \rightarrow$ $\sigma^{*}$ S-H interactions obtained through NBO analysis. Although weak, the $\mathrm{S}-\mathrm{H} \cdots \mathrm{O}=\mathrm{C}$ IHB is proven to play a key role in the conformational preferences of 3-mpa. The detailed results obtained in this study contribute to a better understanding of the characteristics of sulfur HBs.

\section{SUPPLEMENTARY MATERIAL}

Appendix I Equilibrium geometries of the most stable conformers of 3-mpa, 3-hydroxypropionic acid, and thioglycolic acid obtained at the B3LYP-D3BJ/aug-cc-pVTZ

Appendix II Rotational parameters and relative energies for the conformers of 3-mpa calculated at B3LYP, $\omega B$ 97X-D, and M062-X methods with the aug-cc-pVTZ Appendix III Assigned rotational transitions for the parent species of conformer mpa1 


\section{ACKNOWLEDGEMENTS}

The authors acknowledge the Natural Sciences and Engineering Research Council of Canada (NSERC) for funding this research through the Discovery Grant program, and the University of Manitoba for its advanced research computing facility (Grex). W. G. D. P. Silva is also grateful for the financial support provided through the GETS and UM Graduate Fellowship (UMGF) programs through the Faculty of Graduate Studies of the University of Manitoba.

\section{REFERENCES}

[1] S.J. Grabowski, Chem. Rev. 111 (2011) 2597-2625. doi:10.1021/cr800346f.

[2] L.A.H. van Bergen, M. Alonso, A. Palló, L. Nilsson, F. De Proft, J. Messens, Sci. Rep. 6 (2016) 30369. doi:10.1038/srep30369.

[3] H.S. Biswal in: S. Scheiner (Eds), Noncovalent Forces, Springer International Publishing, Switzerland, 2015, pp. 15-24.

[4] V. Rao Mundlapati, S. Ghosh, A. Bhattacherjee, P. Tiwari, H.S. Biswal, J. Phys. Chem. Lett. 6 (2015) 1385-1389. doi:10.1021/acs.jpclett.5b00491.

[5] L.M. Gregoret, S.D. Rader, R.J. Fletterick, F.E. Cohen, Proteins Struct. Funct. Genet. 9 (1991) 99-107. doi:10.1002/prot.340090204.

[6] N.A. Seifert, D.P. Zaleski, C. Pérez, J.L. Neill, B.H. Pate, M. Vallejo-López, A. Lesarri, E.J. Cocinero, F. Castaño, I. Kleiner, Angew. Chemie Int. Ed. 53 (2014) 3210-3213. 
doi:10.1002/anie.201309848.

[7] A.G. Akmeemana, J.M. Kang, R.E. Dorris, R.D. Nelson, A.M. Anderton, R.A. Peebles, S.A. Peebles, N.A. Seifert, B.H. Pate, Phys. Chem. Chem. Phys. 18 (2016) 24290-24298. doi:10.1039/С6CP04737H.

[8] G. Feng, L. Evangelisti, L.B. Favero, J.U. Grabow, Z. Xia, W. Caminati, Phys. Chem. Chem. Phys. 13 (2011) 14092-14096. doi:10.1039/c1cp20751b.

[9] E. Gougoula, C. Medcraft, I. Alkorta, N.R. Walker, A.C. Legon, J. Chem. Phys. 150 (2019) 084307. doi:10.1063/1.5085281.

[10] S. Grimme, M. Steinmetz, Phys. Chem. Chem. Phys. 15 (2013) 16031. doi:10.1039/c3cp52293h.

[11] G.C. Cole, H. Møllendal, J.C. Guillemin, J. Phys. Chem. A. 110 (2006) 9370-9376. doi:10.1021/jp062093y.

[12] L.-H. Xu, Q. Liu, R.D. Suenram, F.J. Lovas, A.R. Hight Walker, J.O. Jensen, A.C. Samuels, J. Mol. Spectrosc. 228 (2004) 243-250. doi:10.1016/j.jms.2004.04.004.

[13] A. Das, P.K. Mandal, F.J. Lovas, C. Medcraft, N.R. Walker, E. Arunan, Angew. Chemie Int. Ed. 57 (2018) 15199-15203. doi:10.1002/anie.201808162.

[14] M. Juanes, A. Lesarri, R. Pinacho, E. Charro, J.E. Rubio, L. Enríquez, M. Jaraíz, Chem. A Eur. J. 24 (2018) 6564-6571. doi:10.1002/chem.201705727.

[15] P. Salgado, T. Visnevschi-Necrasov, R.P. Kiene, I. Azevedo, A.C.S. Rocha, C.M.R. Almeida, C. Magalhães, J. Chromatogr. B. 992 (2015) 103-108. doi:10.1016/j.jchromb.2015.04.008. 
[16] J. Zhuang, X. Zhang, G. Wang, D. Li, W. Yang, T. Li, J. Mater. Chem. 13 (2003) 18531857. doi:10.1039/b303287f.

[17] J. Kang, J.P. Richardson, D. MacMillan, Chem. Commun. 6 (2009) 407-409. doi:10.1039/b815888f.

[18] A. Karlsson, F. Fonnum, D. Malthe-Sørenssen, J. Storm-Mathisen, Biochem. Pharmacol. 23 (1974) 3053-3061. doi:10.1016/0006-2952(74)90281-0.

[19] M. Belcastro, T. Marino, N. Russo, E. Sicilia, J. Phys. Chem. A. 108 (2004) 8407-8410. doi:10.1021/jp047867u.

[20] G.G. Brown, B.C. Dian, K.O. Douglass, S.M. Geyer, S.T. Shipman, B.H. Pate, Rev. Sci. Instrum. 79 (2008) 053103. doi:10.1063/1.2919120.

[21] T.J. Balle, W.H. Flygare, Rev. Sci. Instrum. 52 (1981) 33-45. doi:10.1063/1.1136443.

[22] G. Sedo, J. van Wijngaarden, J. Chem. Phys. 131 (2009) 044303. doi:10.1063/1.3186756.

[23] L. Evangelisti, G. Sedo, J. van Wijngaarden, J. Phys. Chem. A. 115 (2011) 685-690. doi:10.1021/jp1089905.

[24] T.A. Halgren, J. Comput. Chem. 17 (1995) 490-641.

[25] A.D. Becke, J. Chem. Phys. 98 (1993) 5648-5652. doi:10.1063/1.464913.

[26] S. Grimme, S. Ehrlich, L. Goerigk, J. Comput. Chem. 32 (2011) 1456-1465. doi:10.1002/jcc.21759.

[27] A.D. Becke, E.R. Johnson, J. Chem. Phys. 123 (2005) 154101. doi:10.1063/1.2065267.

[28] T.H. Dunning, J. Chem. Phys. 90 (1989) 1007-1023. doi:10.1063/1.456153. 
[29] I. Uriarte, A. Insausti, E.J. Cocinero, A. Jabri, I. Kleiner, H. Mouhib, I. Alkorta, J. Phys. Chem. Lett. 9 (2018) 5906-5914. doi:10.1021/acs.jpclett.8b02339.

[30] C. Møller, M.S. Plesset, Phys. Rev. 46 (1934) 618-622. doi:10.1103/PhysRev.46.618.

[31] Y. Zhao, D.G. Truhlar, Theor. Chem. Acc. 120 (2008) 215-241. doi:10.1007/s00214-0070310-x.

[32] J.-D. Chai, M. Head-Gordon, Phys. Chem. Chem. Phys. 10 (2008) 6615. doi:10.1039/b810189b.

[33] M.J. Frisch, G.W. Trucks, H.B. Schlegel, G.E. Scuseria, M.A. Robb, J.R. Cheeseman, G. Scalmani, V. Barone, G.A. Petersson, H. Nakatsuji, X. Li, M. Caricato, A. V. Marenich, J. Bloino, B.G. Janesko, R. Gomperts, B. Mennucci, H.P. Hratchian, J. V. Ortiz, A.F. Izmaylov, J.L. Sonnenberg, D. Williams-Young, F. Ding, F. Lipparini, F. Egidi, J. Goings, B. Peng, A. Petrone, T. Henderson, D. Ranasinghe, V.G. Zakrzewski, J. Gao, N. Rega, G. Zheng, W. Liang, M. Hada, M. Ehara, K. Toyota, R. Fukuda, J. Hasegawa, M. Ishida, T. Nakajima, Y. Honda, O. Kitao, H. Nakai, T. Vreven, K. Throssell, J. Montgomery, J. A., J.E. Peralta, F. Ogliaro, M.J. Bearpark, J.J. Heyd, E.N. Brothers, K.N. Kudin, V.N. Staroverov, T.A. Keith, R. Kobayashi, J. Normand, K. Raghavachari, A.P. Rendell, J.C. Burant, S.S. Iyengar, J. Tomasi, M. Cossi, J.M. Millam, M. Klene, C. Adamo, R. Cammi, J.W. Ochterski, R.L. Martin, K. Morokuma, O. Farkas, J.B. Foresman, D.J. Fox, Gaussian 16, Revision B.01 Gaussian, Inc., Wallingford, CT, 2016.

[34] R.F.W. Bader, Acc. Chem. Res. 18 (1985) 9-15. doi:10.1021/ar00109a003.

[35] E.R. Johnson, S. Keinan, P. Mori Sánchez, J. Contreras García, A.J. Cohen, W. Yang, J. Am. Chem. Soc. 132 (2010) 6498-6506. doi:10.1021/ja100936w. 
[36] F. Weinhold, C.R. Landis, E.D. Glendening, Int. Rev. Phys. Chem. 35 (2016) 399-440. doi:10.1080/0144235X.2016.1192262.

[37] T.A. Keith, AIMAll (Version 17.11.14), TK Gristmill Software, Overland Park, KS, 2016 (aim.tkgristmill.com).

[38] J. Contreras-García, E.R. Johnson, S. Keinan, R. Chaudret, J.-P. Piquemal, D.N. Beratan, W. Yang, J. Chem. Theory Comput. 7 (2011) 625-632. doi:10.1021/ct100641a.

[39] E.D. Glendening, J.K. Badenhoop, A.E. Reed, J.E. Carpenter, J.A. Bohmann, C.M. Morales, F. Weinhold, NBO 6.0, Theoretical Chemistry Institute, University of Wiscosin, Madison, 2009.

[40] W. Caminati, A. Maris, P.G. Favero, J. Mol. Spectrosc. 175 (1996) 215-216. doi:10.1006/jmsp.1996.0024.

[41] I. Yamaguchi, H. Hasegawa, H. Hirono, Y. Aizawa, M. Takagi, H. Watanabe, T. Sekiguchi, H. Kobayashi, H. Terasawa, K. Watanabe, J. Mol. Spectrosc. 172 (1995) 296-298. doi:10.1006/jmsp.1995.1177.

[42] C. Alemán, J. Casanovas, D. Zanuy, H.K. Hall, J. Org. Chem. 70 (2005) 2950-2956. doi:10.1021/jo0478407.

[43] C.M. Western, J. Quant. Spectrosc. Radiat. Transf. 186 (2017) 221-242. doi:10.1016/j.jqsrt.2016.04.010.

[44] H.M. Pickett, J. Mol. Spectrosc. 148 (1991) 371-377. doi:10.1016/0022-2852(91)90393-O.

[45] J.K.G. Watson, J. Chem. Phys. 48 (1968) 4517-4524. doi:10.1063/1.1668020.

[46] J.L. Alonso, I. Peña, J.C. López, E.R. Alonso, V. Vaquero, Chem. - A Eur. J. 25 (2019) 
2288-2294. doi:10.1002/chem.201805038.

[47] R.S. Ruoff, T.D. Klots, T. Emilsson, H.S. Gutowsky, J. Chem. Phys. 93 (1990) 3142-3150. doi:10.1063/1.458848.

[48] R.F.W. Bader, J. Phys. Chem. A. 5639 (1998) 7314-7323. doi:10.1021/jp981794v.

[49] U. Koch, P.L.A. Popelier, J. Phys. Chem. 99 (1995) 9747-9754. doi:10.1021/j100024a016.

[50] W.G.D.P. Silva, J.M. Silla, R.A. Cormanich, S.A. Fernandes, M.P. Freitas, Chem. Phys. 473 (2016) 17-23. doi:10.1016/j.chemphys.2016.04.018.

[51] L.J. Karas, P.R. Batista, R. V Viesser, C.F. Tormena, R. Rittner, P.R. de Oliveira, Phys. Chem. Chem. Phys. 19 (2017) 16904-16913. doi:10.1039/C7CP03572A.

[52] J.R. Lane, J. Contreras-García, J.P. Piquemal, B.J. Miller, H.G. Kjaergaard, J. Chem. Theory Comput. 9 (2013) 3263-3266. doi:10.1021/ct400420r. 